\title{
Nasal Drops, Solution Dosage Form
}

National Cancer Institute

\section{Source}

National Cancer Institute. Nasal Drops, Solution Dosage Form. NCI Thesaurus. Code C150068.

Liquid sing le-dose or multidose preparation consisting of a solution intended for nasal use by means of a suitable applicator. 